Article

\title{
Discovery of Selective Inhibitor Leads by Targeting an Allosteric Site in Insulin-Regulated Aminopeptidase
}

\author{
Ioannis Temponeras ${ }^{1}$, Lykourgos Chiniadis ${ }^{1}$, Athanasios Papakyriakou ${ }^{1, *(D)}$ and Efstratios Stratikos ${ }^{1,2, *(D)}$ \\ 1 National Centre for Scientific Research Demokritos, Agia Paraskevi, Attikis, 15341 Athens, Greece; \\ john.t13111@windowslive.com (I.T.); lchiniadis@gmail.com (L.C.) \\ 2 Laboratory of Biochemistry, Department of Chemistry, National and Kapodistrian University of Athens, \\ Panepistimioupolis Zografou, 15771 Athens, Greece \\ * Correspondence: thpap@bio.demokritos.gr (A.P.); estratikos@chem.uoa.gr (E.S.)
}

Citation: Temponeras, I.; Chiniadis, L.; Papakyriakou, A.; Stratikos, E.

Discovery of Selective Inhibitor Leads by Targeting an Allosteric Site in Insulin-Regulated Aminopeptidase. Pharmaceuticals 2021, 14, 584. https:// doi.org/10.3390/ph14060584

Academic Editors: Jean Jacques Vanden Eynde and Annie Mayence

Received: 3 June 2021

Accepted: 16 June 2021

Published: 18 June 2021

Publisher's Note: MDPI stays neutral with regard to jurisdictional claims in published maps and institutional affiliations.

Copyright: (c) 2021 by the authors. Licensee MDPI, Basel, Switzerland. This article is an open access article distributed under the terms and conditions of the Creative Commons Attribution (CC BY) license (https:// creativecommons.org/licenses/by/ $4.0 /)$.

\begin{abstract}
Insulin-Regulated aminopeptidase (IRAP) is a zinc-dependent aminopeptidase with several important biological functions and is an emerging pharmaceutical target for cognitive enhancement and immune system regulation. Aiming to discover lead-like IRAP inhibitors with enhanced selectivity versus homologous enzymes, we targeted an allosteric site at the C-terminal domain pocket of IRAP. We compiled a library of 2.5 million commercially available compounds from the ZINC database, and performed molecular docking at the target pocket of IRAP and the corresponding pocket of the homologous endoplasmic reticulum aminopeptidase 1 (ERAP1). Of the top compounds that showed high selectivity, 305 were further analyzed by molecular dynamics simulations and free energy calculations, leading to the selection of 33 compounds for in vitro evaluation. Two orthogonal functional assays were employed: one using a small fluorogenic substrate and one following the degradation of oxytocin, a natural peptidic substrate of IRAP. In vitro evaluation suggested that several of the compounds tested can inhibit IRAP, but the inhibition profile was dependent on substrate size, consistent with the allosteric nature of the targeted site. Overall, our results describe several novel leads as IRAP inhibitors and suggest that the C-terminal domain pocket of IRAP is a promising target for developing highly selective IRAP inhibitors.
\end{abstract}

Keywords: aminopeptidase; peptides; lead-like inhibitors; docking; molecular dynamics; free-energy calculations; enzymatic assays; allosteric; cognitive enhancement; immune system

\section{Introduction}

Insulin-Regulated Aminopeptidase (IRAP, EC 3.4.11.3) is a transmembrane zinc metalloprotease that has been implicated in several important biological functions ranging from roles in glucose metabolism, cognitive functions and the immune system [1-4]. Currently, IRAP is under investigation as a pharmaceutical target for cognitive disorders [5]. Although several IRAP inhibitors have been developed [6-9] no clinical applications have yet been reported. Possible reasons include limited selectivity, limited pharmacodynamics or deficient pharmacokinetics. Up to now, most efforts on developing IRAP inhibitors have either targeted the active site or utilized random library screening [10-12]. However, the IRAP active site is highly homologous to other aminopeptidases of the M1 family and is characterized by structural plasticity that could complicate the development of potent and selective inhibitors [13]. As a result, targeting other sites in IRAP could constitute a viable strategy that would allow the discovery of novel leads that could be developed to inhibitors with superior selectivity and pharmacodynamics. Indeed, in a recent study, a spiro-oxindole dihydroquinazolinone derivative was demonstrated to be an un-competitive inhibitor of IRAP trimming a small dipeptide substrate, although the exact mechanism of inhibition is yet to be determined [12].

The IRAP crystal structure has revealed a large internal cavity, sufficient in size to accommodate large peptidic substrates, adjacent to the catalytic site [14]. An additional 
crystal structure of a complex between a pseudopeptide inhibitor and IRAP revealed a distinct conformation in which the enzyme adopts a closed structure and the internal cavity has no direct access to the outside solvent [13]. A structure with similar configuration, solved at $1.7 \AA$ resolution, has been reported for the homologous aminopeptidase ER aminopeptidase 1 (ERAP1), an enzyme important for the adaptive immune response [15]. In that structure, a similar internal cavity in ERAP1 was found to feature small MW buffer components, a malic acid (MA) and a bis-tris-propane molecule (B3P) bound onto two distinct allosteric sites (Figure 1). The site accommodating the malic acid was later discovered to be the site of an allosteric inhibitor of ERAP1 [16]. Inspired by that finding, we decided to focus on the allosteric site in IRAP that is equivalent to the bis-tris-propane site in ERAP1, in order to discover allosteric inhibitors with enhanced selectivity (Figure 1).

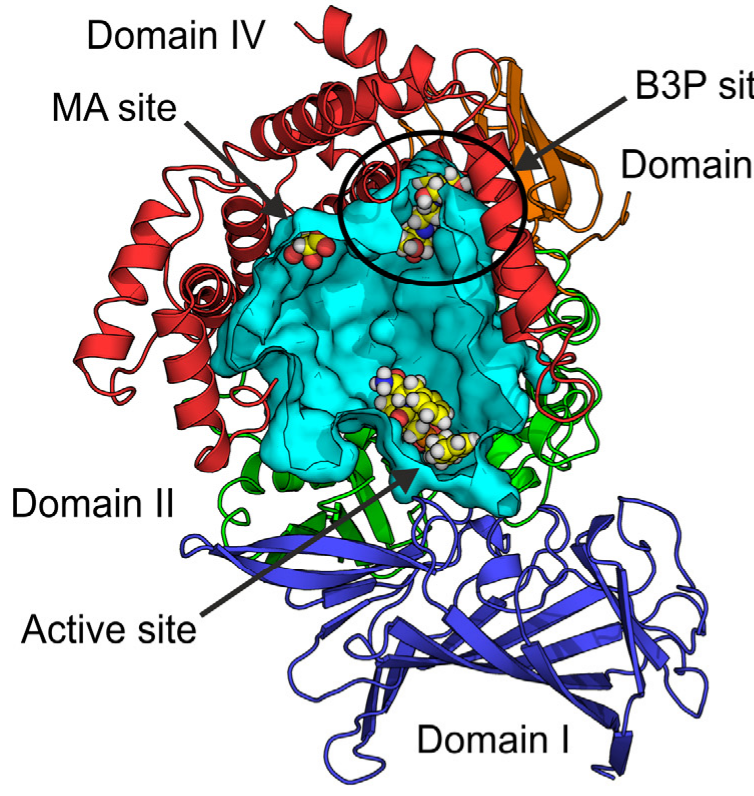

ERAP1

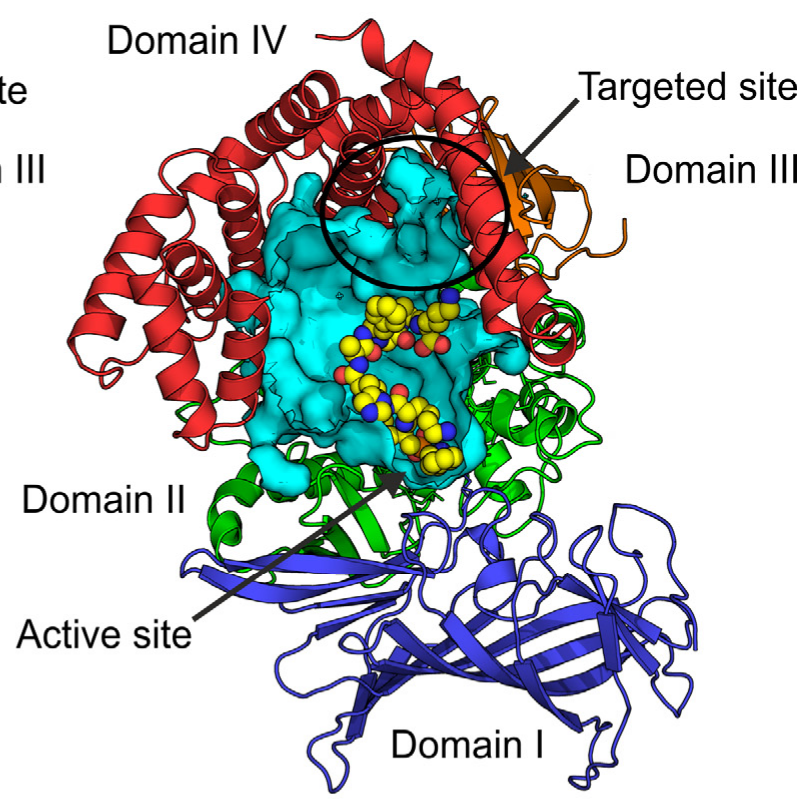

IRAP

Figure 1. Schematic representations of the internal cavity or ERAP1 (left) and IRAP (right). Figures were constructed based on the coordinates of PDB ID: 6q4r and 4z7i, respectively, retrieved from RCSB Protein Data Bank [17]. Internal cavities are shown in cyan surface representation and proteins in cartoon representations are color-coded by domain. Ligands found in each cavity are shown in sphere representation (C: yellow, N: blue, O: red, H: white). The active sites accommodating an inhibitor for ERAP1 and a peptide analogue for IRAP are indicated. The sites where a bis-tris propane (B3P) and a malic acid (MA) molecule were found in ERAP1 are indicated, as well as the equivalent site of IRAP that was targeted for virtual ligand screening.

\section{Results}

\subsection{Selection of a Lead-Like Dataset}

With the aim to discover compounds that bind at the corresponding pocket of IRAP and inhibit its enzymatic activity for natural substrates, we carried out a multi-step virtual screening strategy (Figure 2). First, we obtained a lead-like compilation of compounds from the ZINC15 database [18] by applying commercial availability, reactivity and physiochemical properties filters. The ZINC15 chemical space comprises more than 1.4 billion of annotated substances, of which approximately 14 million 3D protomers (in the $\mathrm{pH}$ range of 6.4-8.4) are readily available for purchase from commercial vendors (lead time of 2 weeks) and display standard reactivity (including mildly reactive electrophilic or nucleophilic groups, http:/ / wiki.docking.org/index.php/Reactivity_axis, accessed on 3 June 2021). From this chemical space, we selected a subset of lead-like compounds that was slightly modified from the predefined ZINC15 "Lead-Like" subset (Figure S1). In particular, a 2.5-million subset was obtained using the following filters: (i) in-stock availability; (ii) refer- 
ence $\mathrm{pH}$ of 7.4; (iii) free from reactive groups or PAINS patterns (anodyne subset) [19]; (iv) MW between 200 and 350; (v) calculated $\log P$ (octanol-water partition coefficient) below 3.0. The lower MW and higher hydrophobicity cut-offs were applied so as to maximize the potential aqueous solubility of the selected compounds (the preset "Lead-Like" subset contains substances with MW $>250$ and $\log P<3.5$ ). The anodyne filter was chosen in order to avoid compounds with reactive groups or assay-interfering patterns, including PAINS, which could react with the enzyme or interfere with the assays employed (vide infra).

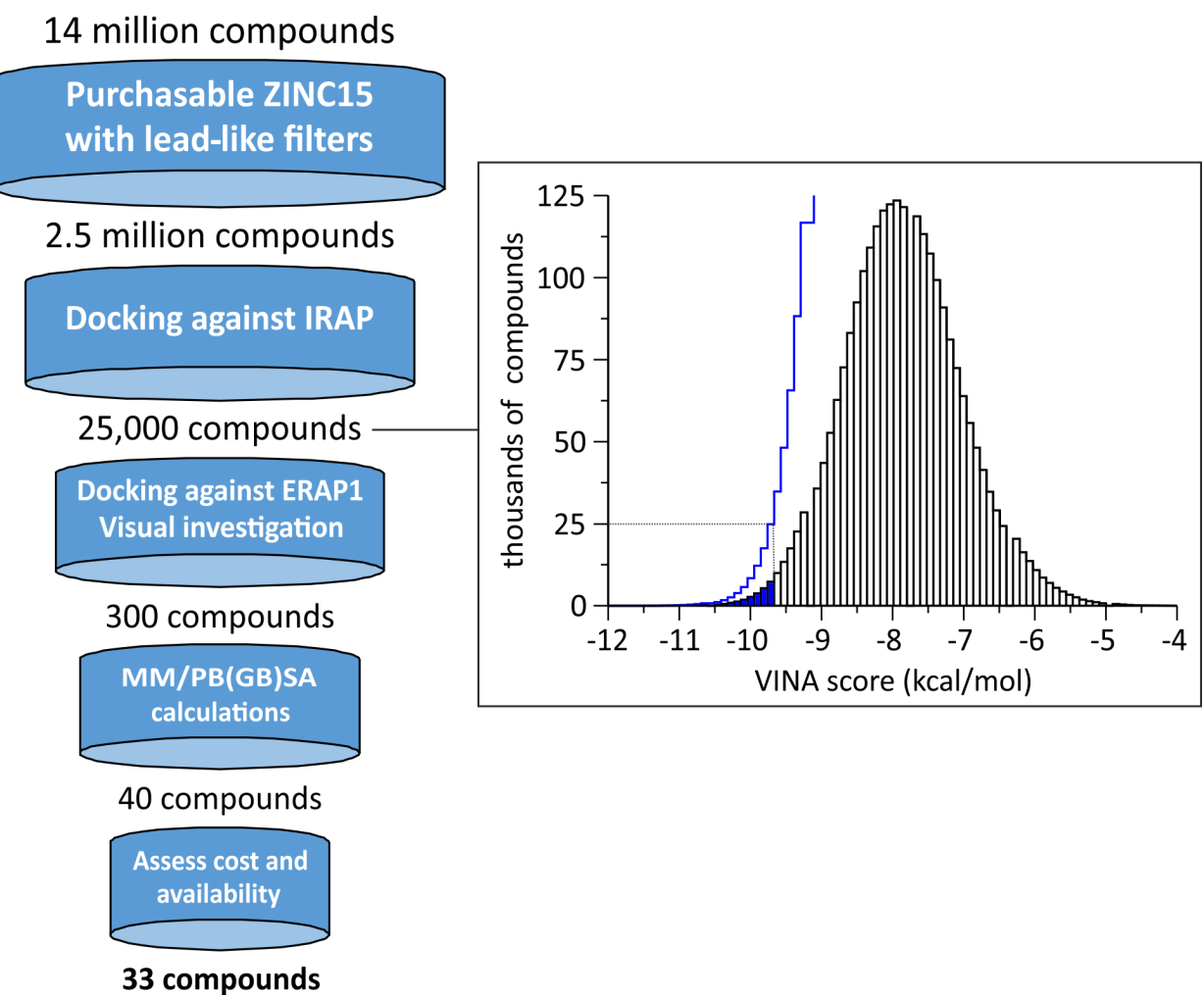

Figure 2. Virtual screening strategy employed for the discovery of lead-like allosteric inhibitors of IRAP, indicating the approximate number of compounds used in each stage. Inset, the distribution of number of compounds (in thousands) as a function of estimated free energy of binding (VINA score). The blue line shows the cumulative distribution and the highlighted region indicates the top $1 \%$ (lowest energy) of the compounds selected.

\subsection{Virtual Screening of the Compounds}

From the selected lead-like subset, a total of ca. 2.45 million compounds were finally obtained in AutoDock PDBQT format [20] and were employed in docking to IRAP using AutoDock VINA [21]. The highest resolution structure of IRAP resolved at $2.5 \AA$ was employed (PDB ID: 5mj6) [13] and the search space was centered within the targeted pocket (see Methods 4.1). The resulting conformers were ranked according to the estimated binding affinity, as given by the VINA score, and from the top-ranked solutions we selected ca. 25,000 compounds for further evaluation (top-1\%, Figure 2). In order to maximize our chances of obtaining IRAP-selective inhibitors, we performed a second round of docking calculations against the corresponding site of ERAP1, the highest homologous enzyme of IRAP within the M1 family of aminopeptidases [22]. The top-ranked subset of compounds identified for IRAP was then employed in docking to ERAP1, using the high-resolution X-ray structure (PDB ID: 6q4r [15]). The resulting VINA scores for ERAP1 were then used to rank the compounds according to their difference in their binding affinities with respect to IRAP, so as to disfavor compounds with high probability to bind ERAP1. In this way, we narrowed down the top-ranked subset to 423 compounds that displayed either binding 
scores below $-10.9 \mathrm{kcal} / \mathrm{mol}$ for IRAP, or a difference in the estimated free energy of binding with respect to ERAP1 below $-2.5 \mathrm{kcal} / \mathrm{mol}$ (Tables S1 and S2, respectively).

\subsection{Free Energy Calculations}

Considering that AutoDock VINA has achieved a standard error of $2.85 \mathrm{kcal} / \mathrm{mol}$ between predicted and experimental free energies of binding [21], and that our selection of compounds displays a range of scores within $2 \mathrm{kcal} / \mathrm{mol}$ (between -11.7 and -9.7 ), we sought to employ a more accurate method for the determination of their relative binding affinity for IRAP. For this reason, we selected MM/PB(GB)SA, a methodology that combines molecular mechanics energies with the Poisson-Boltzmann (or generalized Born) methods and the surface area continuum solvation methods [23]. Although this method is not as accurate for the determination of absolute binding free energies as are more rigorous free energy methods (such as the free energy perturbation and thermodynamic integration methods), $\mathrm{MM} / \mathrm{PB}(\mathrm{GB}) \mathrm{SA}$ is very popular and efficient for the purpose of virtual screening [24]. In particular, we employed the single-trajectory approach by extracting the ensemble average of the free receptor and ligand from a single simulation of the complex. We also omitted calculation of the entropic term, as it has been shown to have minimal effect in comparative results from large sets in virtual screening [25].

Before setting up the MD simulations for the free energy calculations, we performed an additional step comprising visual investigation of the predicted bound conformations for the 423 selected IRAP-ligand complexes, so as to discard compounds that did not display potential for interactions with key residues, or hydrogen-bonding interactions (Figure 3). Specifically, we favored compounds with the potential for interactions with Lys520 from domain II, Phe635 from domain III and the pocket comprising mainly of the hydrophobic residues Phe736, Leu774, Leu1009 and Trp1013 from domain IV. In this way, we selected a final set of 305 compounds for further evaluation with MM/PB(GB)SA calculations, which displayed the most favorable interactions at the hinge domain of IRAP and at the same time had specific interactions with residues of domains II and IV. Each complex was simulated for $20 \mathrm{~ns}$ of unbiased MD run in explicit solvent, for an aggregate of $>6 \mu$ s of simulation time (see Methods 4.2). From the second half of each simulation 1000 equally sampled snapshots were extracted for the free energy calculations using 2 parameter sets of MM/GBSA and 2 sets of MM/PBSA calculations for comparison (see Methods 4.3). The full set of results from the free energy calculations, including energy decomposition, are given in Supplementary Material (Tables S3-S6).

\subsection{Final Selection of the Compounds for Evaluation}

The final assessment for the selection of compounds for experimental screening was mainly based on the free energy results from the MM/PBSA calculations. As a result, 32 compounds were selected among the top-30 ranked results obtained using the two MM/PBSA methods (Table S7). In addition, we selected compounds that displayed highly ranked free energy of binding in MM/GBSA calculations, but also taking into consideration their ranking from MM/PBSA results. This way, an additional set of 8 compounds was selected $(\mathbf{1}, 7, \mathbf{1 2}, \mathbf{1 5}, \mathbf{2 4}, \mathbf{2 8}, 29$ and 33 in Table 1). From the final set of 40 unique compounds, 33 were finally obtained from MolPort after considering their availability and cost (Figures S2 and S3). Compounds were used without further purification and as mixtures of enantiomers or diastereomers, where applicable (Table S7). A complete set of their physiochemical properties was calculated using SwissADME server [26] and is given in Figure S4 and Table S8. 


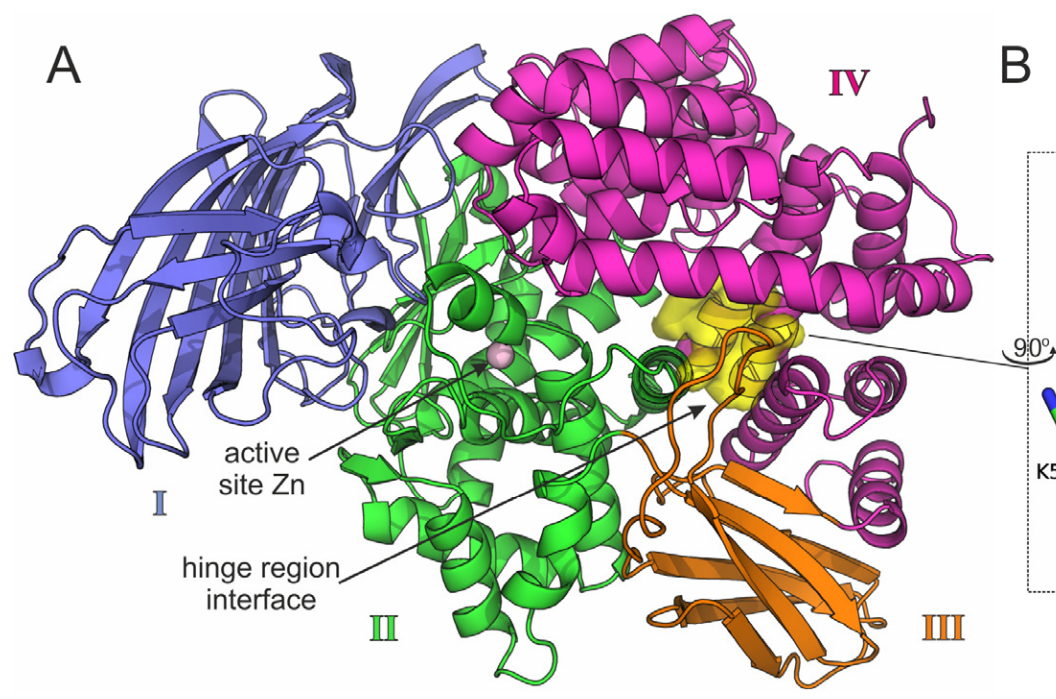

B
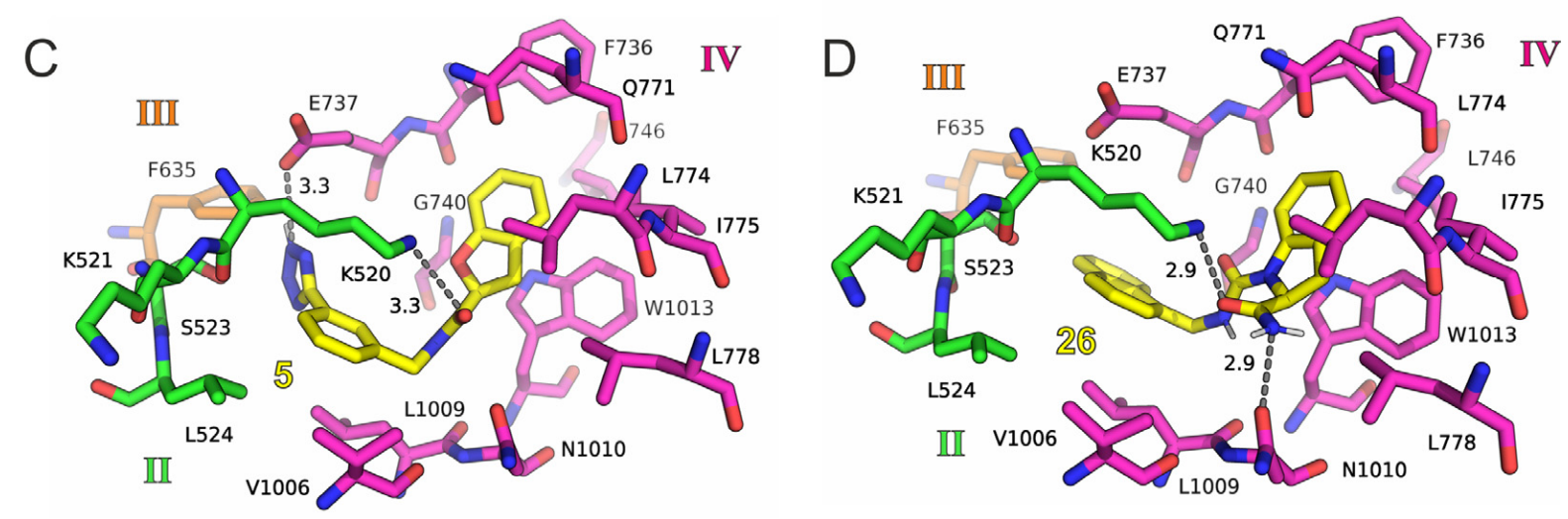

Figure 3. (A) Crystal structure of IRAP illustrating the position of targeted pocket and color-coded according to its functional domain I-IV. (B) Key residues that comprise the targeted pocket, color-coded according to IRAP domain. (C,D) Representative docked poses of two compounds, $\mathbf{5}$ and $\mathbf{2 6}$, shown in yellow-C sticks. Hydrogen bonding interactions with key residues from domains II and IV of IRAP are indicated with dashed lines and the corresponding distance in $\AA$.

Table 1. The final set of 33 compounds obtained for experimental evaluation, indicating docking scores against IRAP and ERAP1, and estimated binding free energy obtained from MM/PBSA calculations using two parameter sets. Energies are in $\mathrm{kcal} / \mathrm{mol}$ and ranking $(\#)$ is relative in each set.

\begin{tabular}{|c|c|c|c|c|c|c|c|}
\hline \multirow{2}{*}{ ID } & \multirow{2}{*}{ MolPort ID } & \multirow{2}{*}{ MW } & \multirow{2}{*}{$\log P^{a}$} & \multicolumn{2}{|c|}{ VINA Score } & \multicolumn{2}{|c|}{$\mathrm{MM} / \mathrm{PBSA} \Delta G_{\text {bind }}$} \\
\hline & & & & IRAP & ERAP1 & PB-1 (Rank) & PB-4 (Rank) \\
\hline 1 & MolPort-027-863-667 & 342.4 & 2.85 & -10.2 & -7.5 & $-1.3(\# 105)$ & $-30.3(\# 59)$ \\
\hline 2 & MolPort-039-039-060 & 331.4 & 2.38 & -11.0 & -9.2 & $-8.1(\# 17)$ & $-31.5(\# 41)$ \\
\hline 3 & MolPort-005-163-536 & 330.4 & 3.45 & -9.9 & -7.1 & $-10.8(\# 6)$ & $-32.6(\# 29)$ \\
\hline 4 & MolPort-027-857-260 & 341.5 & 2.96 & -9.9 & -7.4 & $-7.8(\# 20)$ & $-36.9(\# 3)$ \\
\hline 5 & MolPort-038-425-524 & 335.4 & 2.34 & -11.0 & -8.9 & $-7.5(\# 22)$ & $-33.5(\# 17)$ \\
\hline 6 & MolPort-006-484-933 & 335.4 & 2.71 & -9.9 & -7.4 & $-14.1(\# 2)$ & $-38.7(\# 1)$ \\
\hline 7 & MolPort-007-773-311 & 323.4 & 3.2 & -10.6 & -8.1 & $-5.0(\# 42)$ & $-29.1(\# 78)$ \\
\hline 8 & MolPort-027-906-298 & 332.4 & 2.75 & -10.2 & -7.4 & $-7.4(\# 23)$ & $-32.5(\# 31)$ \\
\hline 9 & MolPort-035-834-132 & 348.4 & 3.07 & -11.1 & -10.0 & $-4.5(\# 51)$ & $-35.4(\# 9)$ \\
\hline 10 & MolPort-020-152-989 & 348.4 & 2.96 & -10.4 & -7.9 & $-9.8(\# 9)$ & $-33.7(\# 14)$ \\
\hline 11 & MolPort-019-674-325 & 338.4 & 2.19 & -11.1 & -8.0 & $-8.0(\# 19)$ & $-32.6(\# 30)$ \\
\hline 12 & MolPort-020-162-189 & 330.4 & 2.15 & -10.9 & -9.5 & $-5.0(\# 43)$ & $-27.2(\# 119)$ \\
\hline 13 & MolPort-028-771-825 & 348.4 & 1.58 & -11.1 & -8.5 & $-6.5(\# 27)$ & $-35.4(\# 8)$ \\
\hline 14 & MolPort-009-543-745 & 326.3 & 2.42 & -10.9 & -8.5 & $-8.0(\# 18)$ & $-29.5(\# 72)$ \\
\hline 15 & MolPort-004-188-947 & 333.5 & 2.97 & -10.1 & -7.1 & $0.3(\# 142)$ & $-26.4(\# 137)$ \\
\hline 16 & MolPort-027-679-372 & 332.4 & 2.77 & -10.9 & -9.5 & $-10.5(\# 8)$ & $-36.2(\# 6)$ \\
\hline
\end{tabular}


Table 1. Cont.

\begin{tabular}{|c|c|c|c|c|c|c|c|}
\hline \multirow{2}{*}{ ID } & \multirow{2}{*}{ MolPort ID } & \multirow{2}{*}{ MW } & \multirow{2}{*}{$\log P^{a}$} & \multicolumn{2}{|c|}{ VINA Score } & \multicolumn{2}{|c|}{ MM/PBSA $\Delta G_{\text {bind }}$} \\
\hline & & & & IRAP & ERAP1 & PB-1 (Rank) & PB-4 (Rank) \\
\hline 17 & MolPort-005-610-771 & 334.3 & 1.59 & -10.9 & -9.6 & $-9.7(\# 10)$ & $-36.0(\# 7)$ \\
\hline 18 & MolPort-009-519-757 & 345.4 & 2.34 & -10.9 & -8.7 & $-5.1(\# 41)$ & $-33.1(\# 22)$ \\
\hline 19 & MolPort-039-259-003 & 322.4 & 1.78 & -10.9 & -8.6 & $-7.0(\# 25)$ & $-32.2(\# 32)$ \\
\hline 20 & MolPort-009-450-517 & 346.4 & 2.62 & -10.9 & -9.2 & $-9.2(\# 12)$ & $-35.1(\# 10)$ \\
\hline 21 & MolPort-004-164-037 & 349.5 & 3.37 & -10.8 & -8.1 & $-7.3(\# 24)$ & $-37.7(\# 2)$ \\
\hline 22 & MolPort-027-704-758 & 324.4 & 1.77 & -10.5 & -8.0 & $-13.9(\# 3)$ & $-36.3(\# 4)$ \\
\hline 23 & MolPort-020-057-841 & 333.4 & 2.94 & -10.9 & -9.2 & $-5.8(\# 33)$ & $-32.9(\# 25)$ \\
\hline 24 & MolPort-009-027-892 & 347.4 & 3.02 & -10.7 & -8.1 & $-3.0(\# 75)$ & $-31.0(\# 46)$ \\
\hline 25 & MolPort-020-053-566 & 338.4 & 2.87 & -9.8 & -7.1 & $-7.7(\# 21)$ & $-33.2(\# 20)$ \\
\hline 26 & MolPort-003-173-337 & 345.4 & 2.34 & -11.0 & -9.7 & $-15.7(\# 1)$ & $-34.3(\# 13)$ \\
\hline 27 & MolPort-003-801-767 & 332.4 & 1.81 & -10.9 & -9.0 & $-8.3(\# 16)$ & $-33.6(\# 15)$ \\
\hline 28 & MolPort-000-832-181 & 346.4 & 2.82 & -10.9 & -9.3 & $-3.9(\# 68)$ & $-30.8(\# 53)$ \\
\hline 29 & MolPort-000-828-576 & 333.4 & 2.72 & -10.9 & -8.3 & $-4.7(\# 50)$ & $-29.3(\# 75)$ \\
\hline 30 & MolPort-000-694-123 & 336.4 & 2.95 & -10.2 & -7.7 & $-9.5(\# 11)$ & $-33.4(\# 18)$ \\
\hline 31 & MolPort-023-297-297 & 336.4 & 2.91 & -10.4 & -7.8 & $-10.5(\# 7)$ & $-34.4(\# 12)$ \\
\hline 32 & MolPort-000-407-697 & 325.4 & 3.47 & -10.1 & -7.6 & $-8.6(\# 14)$ & $-28.5(\# 92)$ \\
\hline 33 & MolPort-001-905-390 & 323.4 & 3.06 & -10.3 & -7.7 & $-4.4(\# 53)$ & $-31.0(\# 50)$ \\
\hline
\end{tabular}

${ }^{a}$ Calculated n-octanol/water partition coefficient using the implicit log P method, iLOGP [27].

\subsection{In Vitro Screening using a Small Fluorigenic Peptide}

To evaluate the inhibitory activity of the selected compounds, we first employed a commonly used kinetic fluorogenic assay that follows hydrolysis of L-Leucine-7-amino4-methylcoumarin [28]. The assay was validated with two previously described IRAP inhibitors, namely DG013A, a pseudopeptide transition state analogue targeting the catalytic site [29], and KE552 (described as compound L-5 in [11]) that was discovered via a library screening and which has an unknown mechanism of inhibition. Their $\mathrm{IC}_{50}$ values of $12 \mathrm{nM}$ for DG013A and 2.1 $\mu \mathrm{M}$ for KE552 (Figure S5) were consistent with published results. Of the 33 compounds tested, 6 resulted in significant dose-dependent inhibition profiles and $\mathrm{IC}_{50}$ values of 50-200 $\mu \mathrm{M}$ (Table 2 and Figure 4). Eight additional compounds showed limited inhibition at the highest concentration tested and were not considered as hits. None of the 6 hit compounds showed any inhibition of the homologous enzyme ERAP1 at concentrations up to $200 \mu \mathrm{M}$ (Figure S6). However, these hits were less selective for ERAP2, with five compounds demonstrating limited inhibition and compound 3 displaying higher potency for ERAP2 than IRAP (Table 2 and Figure S7).

Table 2. $\mathrm{IC}_{50}$ values for compounds 1-33 calculated from the small fluorogenic substrate assays.

\begin{tabular}{|c|c|c|c|c|c|c|c|c|c|c|c|}
\hline \multirow{2}{*}{ ID } & \multicolumn{3}{|c|}{$\mathrm{IC}_{50}(\mu \mathrm{M})$} & \multirow{2}{*}{ ID } & \multicolumn{3}{|c|}{$\mathrm{IC}_{50}(\mu \mathrm{M})$} & \multirow{2}{*}{ ID } & \multicolumn{3}{|c|}{$\mathrm{IC}_{50}(\mu \mathrm{M})$} \\
\hline & IRAP & ERAP1 & ERAP2 & & IRAP & ERAP1 & ERAP2 & & IRAP & ERAP1 & ERAP2 \\
\hline 1 & $>300^{a}$ & - & - & 12 & $N I$ & - & - & 23 & $>300^{a}$ & - & - \\
\hline 2 & NI & - & - & 13 & $192 \pm 21$ & $N I$ & $>300^{a}$ & 24 & $>300^{a}$ & - & - \\
\hline 3 & $192 \pm 35$ & $N I$ & $65 \pm 5$ & 14 & $N I$ & - & - & 25 & $N I$ & - & - \\
\hline 4 & NI & - & - & 15 & $N I$ & - & - & 26 & NI & - & - \\
\hline 5 & $76 \pm 9$ & $N I$ & $>300^{a}$ & 16 & NI & - & - & 27 & NI & - & - \\
\hline 6 & $N I$ & - & - & 17 & $56 \pm 6$ & $N I$ & $\begin{array}{c}280 \pm \\
54\end{array}$ & 28 & $92 \pm 10$ & $N I$ & $>300^{a}$ \\
\hline 7 & $N I$ & - & - & 18 & $>300^{a}$ & - & - & 29 & $N I$ & - & - \\
\hline 8 & NI & - & - & 19 & $>300^{a}$ & - & - & 30 & NI & - & - \\
\hline 9 & $N I$ & - & - & 20 & $>300^{a}$ & - & - & 31 & $194 \pm 24$ & $N I$ & $>300^{a}$ \\
\hline 10 & NI & - & - & 21 & $N I$ & - & - & 32 & NI & - & - \\
\hline 11 & $>300^{a}$ & - & - & 22 & $>300^{a}$ & - & - & 33 & NI & - & - \\
\hline
\end{tabular}

${ }^{a}$ Limited inhibition observed only at the highest concentration tested; NI: no inhibition; (-) not tested. 

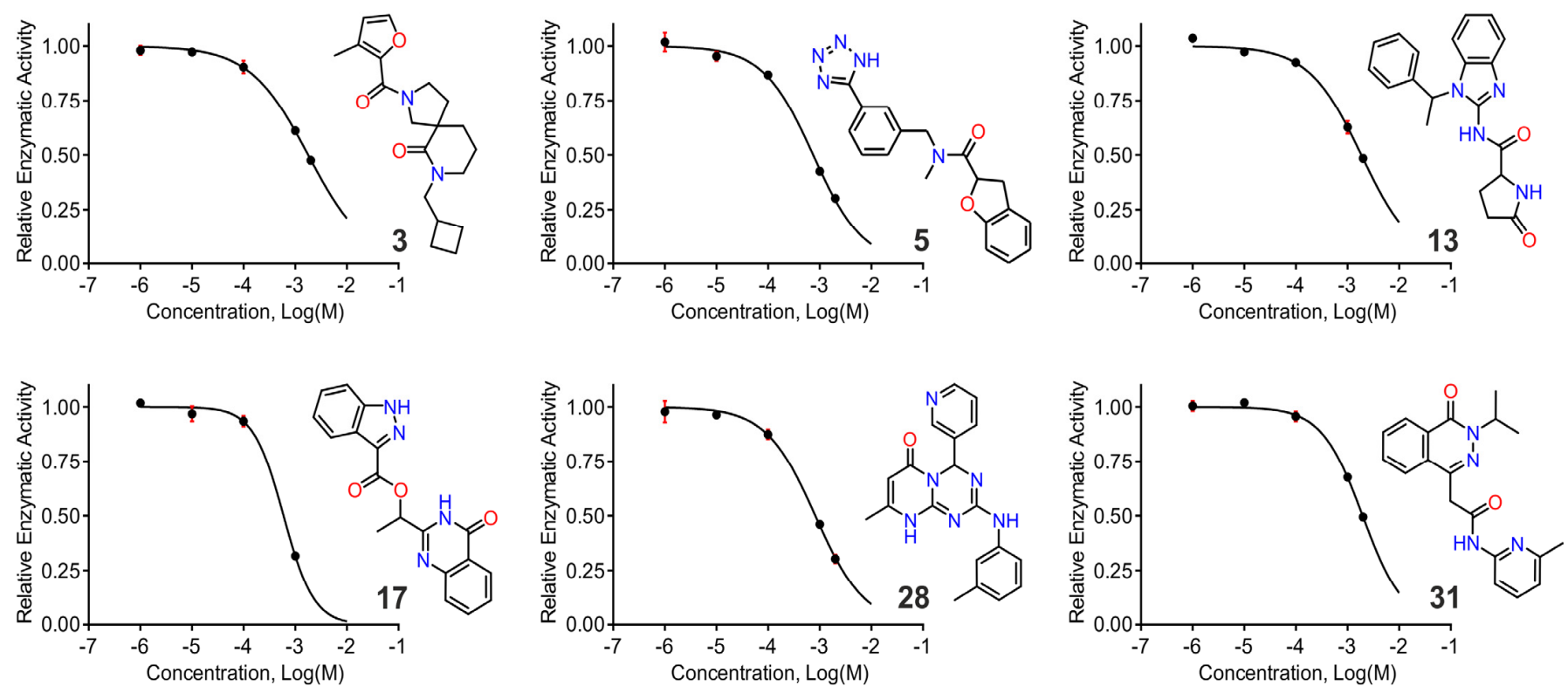

Figure 4. Representative titrations showing the dose-dependent decrease in hydrolysis of the small substrate Leu-AMC by IRAP upon addition of compounds. The structure of the corresponding compound is indicated in each panel and error bars indicate standard deviation that is only shown if significantly larger than the data point size. Solid lines represent fits to a variable-slope inhibitor vs response model using Graphpad prism ${ }^{\mathrm{TM}}$.

\subsection{Inhibition of Oxytocin Trimming}

Although small substrates are a useful tool for characterization of inhibitors of aminopeptidases, the allosteric nature of the targeted site and the complex mechanism employed by IRAP [30] prompted us to also characterize the activity of the compounds in inhibiting the hydrolysis of a physiological substrate of IRAP, oxytocin [31]. The cleavage of the N-terminal residue of the cyclic nonapeptide oxytocin can be followed by highperformance liquid chromatography on a C18 reversed phase column (Figure 5A). This assay was validated using DG013A and KE552 as described above for the Leu-AMC substrate. DG013A was able to completely inhibit oxytocin trimming by IRAP when used at a concentration of $1 \mu \mathrm{M}$ as expected by its calculated $\mathrm{IC}_{50}$ for Leu-AMC. Remarkably, KE552 was less effective against oxytocin and inhibited only $10 \%$ of trimming at $10 \mu \mathrm{M}$ and up to $38 \%$ when used at $100 \mu \mathrm{M}$. The $\%$ inhibition of IRAP activity for each compound tested is shown in Table 3. The most active compound was found to be 26, which was, surprisingly, not active in inhibiting the hydrolysis of Leu-AMC by IRAP and ERAP1, nor the hydrolysis of Arg-AMC by ERAP2 (Figure 5B and Figure S8). The ability of 26 to inhibit the processing of oxytocin was found to be dose-dependent with $\mathrm{IC}_{50}$ of $41 \pm 2 \mu \mathrm{M}$ (Figure 5C).

\subsection{Evaluation of Cellular Toxicity}

Given the interesting properties of compound 26, we evaluated its toxicity against cultured cells. MOLT4 cells were incubated with 26 at concentrations up to $200 \mu \mathrm{M}$ for $48 \mathrm{~h}$ and their viability was assessed by the standard 3-(4,5-dimethylthiazol-2-yl)-2,5diphenyltetrazolium bromide (MTT) assay [32]. No apparent toxicity was observed up to $200 \mu \mathrm{M}$ (Figure 5D). 

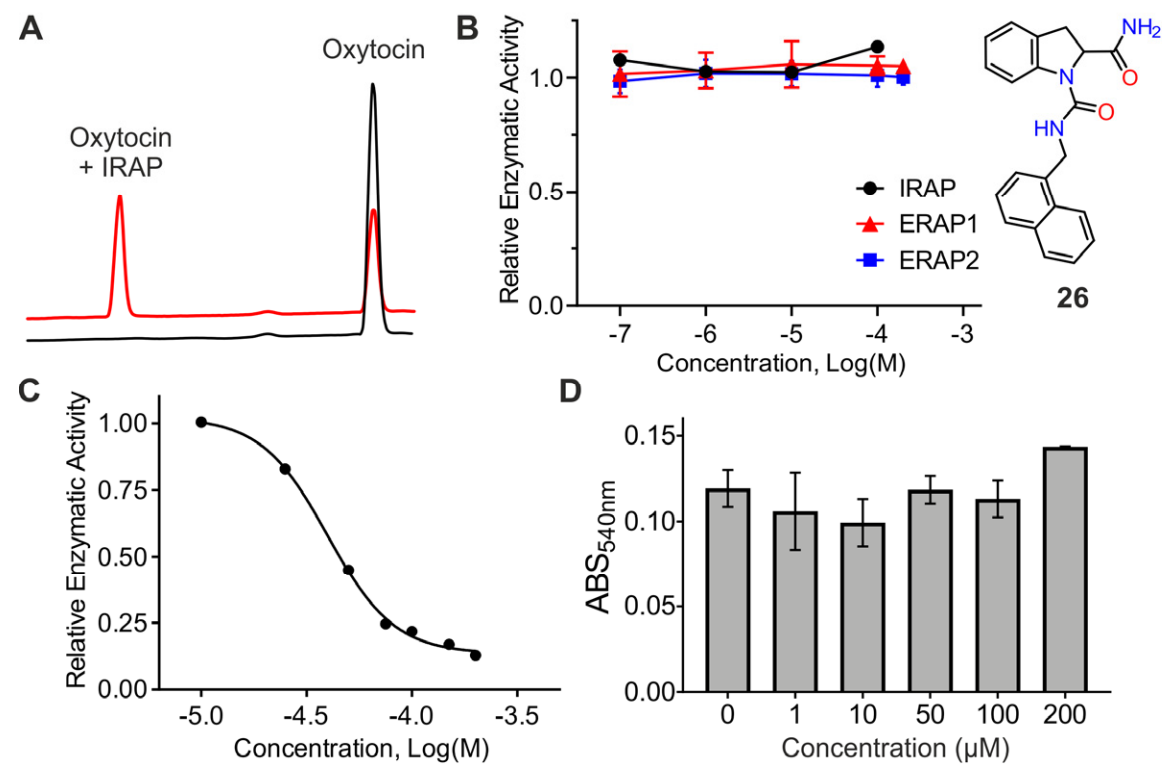

D

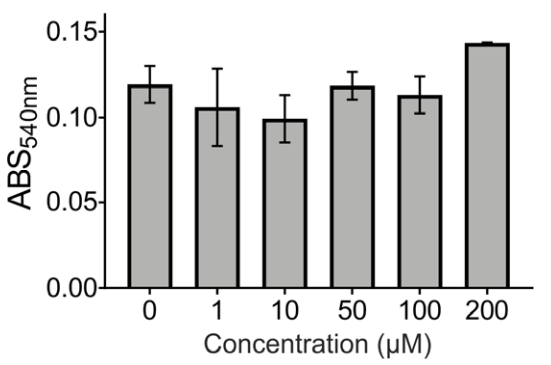

Figure 5. (A) Representative chromatograms of trimming of oxytocin by IRAP as monitored by HPLC. (B) Effect of compound 26 (inset) on the hydrolysis of $50 \mu \mathrm{M}$ Leu-AMC by IRAP and ERAP1, and on the hydrolysis of $50 \mu \mathrm{M}$ Arg-AMC by ERAP2. (C) Dose-dependent inhibition of IRAP trimming of oxytocin by compound 26. (D) Evaluation of the cellular toxicity of $\mathbf{2 6}$ using the MTT assay.

Table 3. Inhibition of oxytocin trimming by IRAP in the presence of 1-33 and the two known inhibitors, DG013A and KE552, at a concentration of $100 \mu \mathrm{M}$.

\begin{tabular}{cccccc}
\hline ID & \% Inhibition & ID & \% Inhibition & ID & \% Inhibition \\
\hline $\mathbf{1}$ & 0 & $\mathbf{1 2}$ & 44 & $\mathbf{2 3}$ & 26 \\
$\mathbf{2}$ & 26 & $\mathbf{1 3}$ & 10 & $\mathbf{2 4}$ & 0 \\
$\mathbf{3}$ & 7 & $\mathbf{1 4}$ & 25 & $\mathbf{2 5}$ & 14 \\
$\mathbf{4}$ & 11 & $\mathbf{1 5}$ & 9 & $\mathbf{2 6}$ & 69 \\
$\mathbf{5}$ & 17 & $\mathbf{1 6}$ & 32 & $\mathbf{2 7}$ & 32 \\
$\mathbf{6}$ & 15 & $\mathbf{1 7}$ & 23 & $\mathbf{2 8}$ & $n . d$. \\
$\mathbf{7}$ & 0 & $\mathbf{1 8}$ & 0 & $\mathbf{2 9}$ & 39 \\
$\mathbf{8}$ & 0 & $\mathbf{1 9}$ & 0 & $\mathbf{3 0}$ & 22 \\
$\mathbf{9}$ & 30 & $\mathbf{2 0}$ & 34 & $\mathbf{3 1}$ & 10 \\
$\mathbf{1 0}$ & 0 & $\mathbf{2 1}$ & 14 & $\mathbf{3 2}$ & 28 \\
$\mathbf{1 1}$ & 13 & $\mathbf{2 2}$ & 0 & $\mathbf{3 3}$ & 10 \\
KE552 & 38 & KE552 $^{a}$ & 10 & DG013A $^{b}$ & 100 \\
\hline
\end{tabular}

n.d. not determined due to peak overlap; ${ }^{a}$ KE552 also tested at $10 \mu \mathrm{M} ;{ }^{b}$ DG013A tested at $1 \mu \mathrm{M}$.

\section{Discussion}

Targeting allosteric sites of enzymes in order to develop inhibitors can be challenging since binding of the compound does not directly compete with binding of the substrate at the catalytic site. At the same time, the conserved nature of many enzymatic active sites, while it can empower rational design, can make achievement of high degrees of selectivity difficult. This has been reported previously for IRAP using phosphinic pseudopeptides that act as transition-state analogues and which are potent inhibitors, but often target homologous aminopeptidases such as ERAP1 and ERAP2 [29]. To circumvent this problem, we targeted an allosteric site in IRAP that has been shown to be able to bind small molecules in the homologous ERAP1 [15]. By combining virtual docking with molecular dynamics and free energy calculations, we were able to identify at least 5 compounds that are selective inhibitors of IRAP with $\mu \mathrm{M}$ potencies and could thus serve as leads for the development of more potent inhibitors (Table 2 and Figure 4). 
One surprising result from our study is the different behavior of some of the hit compounds when using different substrates. Of the 6 most potent compounds that were validated with the small substrate Leu-AMC of IRAP, 5 were able to display some inhibition of oxytocin (Table 3). However, several more compounds that were not able to inhibit Leu-AMC, did display appreciable inhibition of oxytocin cleavage. One notable such case, compound 26, was inactive as an inhibitor of Leu-AMC but inhibited oxytocin cleavage with an $\mathrm{IC}_{50}$ of $41 \mu \mathrm{M}$. A previously identified inhibitor, KE552, demonstrated an apparently opposite behavior, being a good inhibitor of Leu-AMC and a poor inhibitor of oxytocin. This apparent dissociation of inhibitory behavior for different size substrates may be due to the relatively low affinity of these compounds which hinders accurate comparisons. Alternatively, the position of binding away from the active site could underlie such phenomena. Modulation of cleavage of the small substrate would have to proceed allosterically through unknown mechanisms. Such a case has been described for the homologous ERAP1, whose activity can be modulated in a substrate-dependent manner by a small compound binding in the malic acid site, by an allosteric/conformational mechanism [16]. Indeed, a spiro-oxindole dihydroquinazolinone derivative was recently demonstrated to be an uncompetitive inhibitor of IRAP and an allosteric binding site adjacent to the zinc catalytic site was proposed [12]. However, inhibition of oxytocin, a substrate that is large enough to reach towards the allosteric site, could also proceed by a more competitive-like mechanism. It should be noted that the only structure of a peptidic substrate with IRAP known is a structure with a linear peptide analogue that does not appear to occupy the allosteric site targeted here (Figure 1) [14]. However, oxytocin, being a cyclic peptide, could bind in a different conformation and at least partially compete with the targeted site. KE552 binding in a distinct site that does not overlap with oxytocin, could underlie its reduced effectiveness. Additional structural studies will be required to clarify the mechanism of inhibition by these compounds.

Although deciphering the exact mechanism of inhibition by allosteric compounds can be difficult without structural information, some insight can be drawn from the known conformational behavior of IRAP and homologous enzymes. IRAP has been crystalized in two distinct conformations in which domains I/II move in relation to domain IV around the hinge domain III and either form an open configuration that exposes the internal cavity to the solvent or a closed configuration that excludes solvent access to the internal cavity $[13,14]$. Structural analysis using small-angle X-ray scattering has suggested that IRAP in solution is in an open conformation and closes upon inhibitor or substrate binding [33]. As a result, induction of the closed conformation, a conformation that would block substrate-product exchange has been proposed as a possible mechanism of inhibition and could also be the mechanism at work here. A similar mechanism has been proposed for an allosteric inhibitor of ERAP1 [16].

In summary, we describe a virtual docking approach that targets an allosteric site in IRAP and the discovery of several novel and selective IRAP inhibitor leads. Our results suggest that targeting this allosteric site in IRAP is a viable alternative approach for the development of IRAP inhibitors with potential for pharmaceutical applications in cognitive enhancement and the modulation of immune responses.

\section{Materials and Methods}

\subsection{Docking Calculations}

The high-resolution X-ray crystal structures of IRAP in complex with a potent inhibitor (PDB ID: 5mj6) [13] and ERAP1 in complex with a phosphinic pseudopeptide inhibitor (PDB ID: 6q4r) [15] were retrieved from the Protein Data Bank. After removing all heteroatoms, including the inhibitors, their coordinates were superimposed with respect to the domain IV residues comprising a major part of the target pockets. Missing atoms were added using Modeller v9.10 [34] and the $\mathrm{H}++$ server was used to assign protonation states of the titratable groups and add hydrogen atoms accordingly [35]. After removing the non-polar hydrogens, Gasteiger charges and atom types were assigned using AutoDockTools [36]. 
The search space was defined by a $27-\AA$ cube that was centered within the targeted pocket $((x, y, z)=(5,-54,-23)$ with reference to PDB ID: 5mj6). AutoDock VINA [21] was used for docking of c.a. 2.45 million lead-like compounds to IRAP, and its top-ranked subset of 24,842 compounds to ERAP1. The default parameters of VINA were retained except for the exhaustiveness level that was increased to 20. The 3D structure of the compounds were obtained from the ZINC15 database [18] in AutoDock PDBQT format and were used without further optimization. Calculations were performed at the HPC facility "ARIS" of the Hellenic National Infrastructures for Research and Technology, GRNeT, which is kindly acknowledged for the allocated time.

\subsection{Molecular Dynamics Simulations}

Molecular dynamics calculations in explicit solvent were performed for 305 complexes of IRAP with selected, top-ranked compounds using the GPU-accelerated version of PMEMD module in AMBER 18 [37,38]. The AMBER ff14SB force field [39] was used for IRAP and the gaff2 force field [40] for the ligands, for which atom types and AM1-BCC atomic charges [41] were generated using the ANTECHAMBER module in AMBER. For the metal we employed a simple force field with covalent bonds for tetrahedral Zn(II) [13], after replacing the inhibitor with a water molecule. The complexes were immersed into truncated octahedral solvent boxed of pre-equilibrated TIP3P water molecules with a minimum buffer of $10 \AA$ around the complex, and the required number of counter ions was added to obtain charge neutralization of the systems using the LEaP module in AMBER. The equilibration and production protocol employed was similar to that described in our previous works $[13,42,43]$. Briefly, a 1-ns equilibration period was followed by $20 \mathrm{~ns}$ of unrestrained production runs in the isothermal-isobaric (NPT) ensemble. For the free energy calculations 1000 snapshots were equally sampled from the last $10 \mathrm{~ns}$ of the trajectories using the CPPTRAJ module in AMBER [44]. Explicit solvent, counter ions and the metal were removed for the implicit PBSA or GBSA solvent models to be used in the estimation of the solvation energies.

\subsection{Free energy Calculations}

According to the MM/PBSA and MM/GBSA approaches $[23,45,46]$ the binding free energy $\left(\Delta G_{\text {bind }}\right)$ of a small ligand (L) bound to a protein $(\mathrm{P})$ is typically estimated by the ensemble average of the free protein and ligand extracted from a single simulation of their complex (PL):

$$
\Delta G_{\text {bind }}=\left\langle G_{\mathrm{PL}}-G_{\mathrm{P}}-G_{\mathrm{L}}\right\rangle
$$

The free energy of each state is estimated from:

$$
G=E_{\mathrm{bond}}+E_{\mathrm{el}}+E_{\mathrm{vdW}}+G_{\mathrm{pol}}+G_{\mathrm{np}}-T S
$$

where the first three terms are the molecular mechanics energy terms from bonded ( $\left.E_{\mathrm{bond}}\right)$, electrostatic interactions $\left(E_{\mathrm{el}}\right)$ and van der Waals interactions $\left(E_{\mathrm{vdW}}\right)$, followed by the polar and non-polar contributions to the solvation free energy ( $G_{\mathrm{pol}}$ and $G_{\mathrm{np}}$, respectively). The last term represents the entropic contribution to the free energy of binding (the absolute temperature $T$ multiplied by the entropy $S$ ) and is often estimated by normal-mode analysis [47]. Considering the high computational cost of the entropy calculations and given that we employed the free energy calculations for a large dataset comparatively, this term was omitted as suggested by others [48].

The polar solvation term $G_{\text {pol }}$ in Equation (2) is calculated by applying an implicit continuum solvent and using a finite-difference solution or a Generalized Born (GB) pairwise approximation of the Poisson-Boltzmann equation (PB) [24]. The non-polar solvation free energy contribution to the solvation free energy $\left(G_{n p}\right.$ in Equation (2)) can be simply estimated as being proportional to the solvent accessible surface area (SASA) of the solute:

$$
\Delta G_{\mathrm{np}}=\gamma * \mathrm{SASA}+\mathrm{b}
$$


where the surface tension $\gamma$ and the correction term $b$ are given constant values for all solute molecules. In more recent approaches [49] the solute cavity formation and the van der Waals interactions free energy terms are estimated as separate terms:

$$
\Delta G_{\mathrm{np}}=\Delta G_{\mathrm{disp}}+\gamma * \mathrm{SASA}+\mathrm{b}
$$

where the dispersion term $\left(\Delta G_{\text {disp }}\right)$ is often computed using a solvent accessible surface, or a solvent accessible volume integration, and the scaling factors $\gamma$ and $b$ are adjusted according to the choice of the atomic and solvent probe radii employed (see Table 4 below).

Table 4. Summary of the parameter sets employed in free energy calculations, indicating the method used for calculation of the polar $\left(G_{\text {pol }}\right)$ and the non-polar solvation energy $\left(G_{n p}\right)$ terms.

\begin{tabular}{|c|c|c|c|c|c|c|}
\hline Alias & $G_{\text {pol }}$ Method & Atomic Radii & $G_{\text {np }}$ Method & SASA $^{c}$ & $\gamma$ & b \\
\hline$P B-1$ & $\operatorname{PBSA}^{a}$ & TL-mbondi ${ }^{b}$ & Equation (4) & $\mathrm{PBSA}^{d}$ & 0.03780 & -0.5692 \\
\hline$P B-4$ & $\operatorname{PBSA}^{a}$ & mbondi [50] & Equation (3) & Molsurf [51] & 0.00720 & 0.0000 \\
\hline$G B-1$ & $\mathrm{~GB}^{\mathrm{HTC}}$ [52] & mbondi [50] & Equation (3) & LCPO [53] & 0.00720 & 0.0000 \\
\hline$G B-5$ & $\mathrm{~GB}^{\mathrm{OTC}-\mathrm{II}}[54]$ & mbondi2 [54] & Equation (3) & LCPO [53] & 0.00500 & 0.0000 \\
\hline
\end{tabular}

${ }^{a}$ Poisson-Boltzmann solver PICCG as implemented in the PBSA module of AMBER $18 .{ }^{b}$ Radii for protein atoms as optimized by Tan \& Luo [55], with mbondi radii for the ligand [50]. ${ }^{c}$ Method or program used for the calculation of the solvent-accessible surface area. ${ }^{d} \Delta G_{\text {disp }}$ calculated by a numerical determination of the solvent accessible surface area [49].

In this work, we employed two MM/PBSA and two MM/GBSA parameter sets designated as $P B-1$ and $P B-4$ for the MM/PBSA, and GB- 1 and GB-5 for the MM/GBSA approach (Table 4), in reference to AMBER 18 manual (https:/ / ambermd.org/doc12/Amber18.pdf; accessed on 3 June 2021). Post-processing of the snapshots taken from the MD trajectories was performed with the MPI version of the MMPBSA python script in AMBER 18. Selection of the compounds for experimental testing was mainly based on the free energy results of MM/PBSA method PB-1, followed by a selection of highly ranked compounds as given by $P B-4$ and the two MM/GBSA methods, in conjunction with their relative ranking in all four methods. The free energy results and energy decomposition for the 305 compounds using the four methods are provided within the Supplementary Material (Tables S3-S6) and a summary of the results obtained for the 33 selected compounds shown in Figure S2 is given in Table S7.

\subsection{Protein Production and Purification}

The extracellular domain of human IRAP was isolated in recombinant form from stably transfected HEK $293 \mathrm{~S} \mathrm{GnTI}^{(-)}$cells as described previously [14]. Human recombinant ERAP1 and ERAP2 was isolated from Hi5 cells infected by baculovirus as described previously $[42,56]$.

\subsection{Enzymatic Assays}

Enzymatic assays using the substrate L-Leucine-7-amido-4-coumarin (Sigma, St. Louis, MO, USA) or L-Arginine-7-amido-4-coumarin (Sigma) were performed as described previously [42]. Oxytocin cleavage by IRAP was followed by high-performance liquid chromatography. Briefly, oxytocin $(40 \mu \mathrm{M})$ was incubated with recombinant IRAP $(2-200 \mathrm{nM})$ at $37^{\circ} \mathrm{C}$ for $30 \mathrm{~min}$ and the reaction was terminated by adding $0.25 \%(v / v)$ trifluoroacetic acid (TFA) and kept at $-80{ }^{\circ} \mathrm{C}$ until analysis. The reactions were analyzed using a reversed phase C18 column (chromolith C-18 column, Merck, Kenilworth, NJ, USA) by following the absorbance at $220 \mathrm{~nm}$ using a linear gradient (solvent A: 0.05\% TFA, 10\% acetonitrile, solvent B: $0.05 \%$ TFA, $40 \%$ acetonitrile). The percentage of substrate cleaved was calculated by integrating the surface of the substrate and product peaks.

\subsection{Inhibitors}

Compound KE552 (described as compound L-5 in [11]) was generously provided by Dr. Mathias Hallberg (Uppsala University, Sweden). Compound DG013A (described 
in [29]) was generously provided by Dr. Dimitris Georgiadis (National and Kapodistrian University of Athens, Greece). Compounds 1-33 were purchased from MolPort and were used without further purification, as mixtures of enantiomers or diastereomers where applicable (Table S7).

\subsection{Cellular Toxicity Assay}

MOLT4 cells (CRL-1582 from ATCC, 5000 cells/well) were cultured in $100 \mu \mathrm{L}$ RPMI 1640 supplemented with $2 \mathrm{mM}$ glutamine, 10\% heat-inactivated FBS (Gibco), 1\% penicillin and streptomycin and incubated at $37{ }^{\circ} \mathrm{C}, 5 \% \mathrm{CO}_{2}$ in the presence of varying concentrations of compound $26(0-200 \mu \mathrm{M})$ for $48 \mathrm{~h}$. After the incubation, $100 \mu \mathrm{L}$ of RPMI 1640 containing $2 \mathrm{mg} / \mathrm{mL}$ 3-(4,5-dimethylthiazol-2-yl)-2,5-diphenyltetrazolium bromide (MTT reagent) were added to each well. The cells were allowed to rest for $4 \mathrm{~h}$ and then centrifuged at $1250 \mathrm{rpm}$ for $5 \mathrm{~min}$ at room temperature. The resulting formazan crystals were dissolved in DMSO $(100 \mu \mathrm{L})$ and the absorbance intensity measured on a TECAN infinite M200 microplate fluorescence reader at $540 \mathrm{~nm}$ with reference at $620 \mathrm{~nm}$. All experiments were performed in triplicate.

Supplementary Materials: The following are available online at https://www.mdpi.com/article/ 10.3390/ph14060584/s1, Detailed computational results (Figures S1-S4 and Tables S1-S8), and supplementary experimental results in Figures S5-S8.

Author Contributions: Conceptualization, A.P. and E.S.; methodology, A.P., E.S., I.T. and L.C.; investigation, A.P., E.S., I.T. and L.C.; data curation, I.T. and L.C; writing- original draft preparation, E.S. and A.P.; writing-review and editing, I.T. and L.C.; supervision, E.S. and A.P; All authors have read and agreed to the published version of the manuscript.

Funding: This research is co-financed by Greece and the European Union (European Social FundESF) through the Operational Programme «Human Resources Development, Education and Lifelong Learning 2014-2020» in the context of the project "Structure-based discovery of modulatory molecules for the action of aminopeptidase IRAP" (MIS: 5047831).

Informed Consent Statement: Not applicable.

Data Availability Statement: The data presented in this study are available on reasonable request from the corresponding authors.

Acknowledgments: This work was supported by computational time granted from the National Infrastructures for Research and Technology S.A. (GRNET) in the National HPC facility-ARISunder project ID 'ALLINIRAP'.

Conflicts of Interest: The authors declare no conflict of interest.

\section{References}

1. Keller, S.R. The insulin-regulated aminopeptidase: A companion and regulator of GLUT4. Front Biosci. 2003, 8, s410-s420. [CrossRef]

2. Saveanu, L. IRAP Identifies an Endosomal Compartment Required for MHC Class I Cross-Presentation. Science 2009, 325, $213-217$. [CrossRef]

3. Albiston, A.L.; Morton, C.J.; Ng, H.L.; Pham, V.; Yeatman, H.R.; Ye, S.; Fernando, R.N.; De Bundel, D.; Ascher, D.B.; Mendelsohn, F.A.; et al. Identification and characterization of a new cognitive enhancer based on inhibition of insulin-regulated aminopeptidase. Faseb. J. 2008, 22, 4209-4217. [CrossRef]

4. Weimershaus, M.; Mauvais, F.X.; Evnouchidou, I.; Lawand, M.; Saveanu, L.; van Endert, P. IRAP Endosomes Control Phagosomal Maturation in Dendritic Cells. Front. Cell Dev. Biol. 2020, 8. [CrossRef]

5. Chai, S.Y.; Yeatman, H.R.; Parker, M.W.; Ascher, D.B.; Thompson, P.E.; Mulvey, H.T.; Albiston, A.L. Development of cognitive enhancers based on inhibition of insulin-regulated aminopeptidase. BMC Neurosci. 2008, 9 (Suppl. 2), S14. [CrossRef] [PubMed]

6. Georgiadis, D.; Ziotopoulou, A.; Kaloumenou, E.; Lelis, A.; Papasava, A. The Discovery of Insulin-Regulated Aminopeptidase IRAP) Inhibitors: A Literature Review. Front. Pharmacol. 2020, 11, 1-8. [CrossRef] [PubMed]

7. Barlow, N.; Vanga, S.R.; Sävmarker, J.; Sandström, A.; Burns, P.; Hallberg, A.; Åqvist, J.; Gutiérrez-de-Terán, H.; Hallberg, M.; Larhed, M.; et al. Macrocyclic peptidomimetics as inhibitors of insulin-regulated aminopeptidase (IRAP). RSC Med. Chem. 2020, 11, 234-244. [CrossRef] [PubMed] 
8. Barlow, N.; Thompson, P.E. IRAP Inhibitors: M1-Aminopeptidase Family Inspiration. Front. Pharmacol. 2020, 11. [CrossRef] [PubMed]

9. Hallberg, M.; Larhed, M. From Angiotensin IV to Small Peptidemimetics Inhibiting Insulin-Regulated Aminopeptidase. Front. Pharmacol. 2020, 11. [CrossRef] [PubMed]

10. Andersson, H.; Demaegdt, H.; Vauquelin, G.; Lindeberg, G.; Karlén, A.; Hallberg, M.; Erdélyi, M.; Hallberg, A. Disulfide cyclized tripeptide analogues of angiotensin IV as potent and selective inhibitors of insulin-regulated aminopeptidase (IRAP). J. Med. Chem. 2010, 53, 8059-8071. [CrossRef]

11. Engen, K.; Rosenström, U.; Axelsson, H.; Konda, V.; Dahllund, L.; Otrocka, M.; Sigmundsson, K.; Nikolaou, A.; Vauquelin, G.; Hallberg, M.; et al. Identification of Drug-Like Inhibitors of Insulin-Regulated Aminopeptidase Through Small-Molecule Screening. Assay Drug Dev. Technol. 2016, 14, 180-193. [CrossRef]

12. Engen, K.; Vanga, S.R.; Lundbäck, T.; Agalo, F.; Konda, V.; Jensen, A.J.; Åqvist, J.; Gutiérrez-de-Terán, H.; Hallberg, M.; Larhed, M.; et al. Synthesis, Evaluation and Proposed Binding Pose of Substituted Spiro-Oxindole Dihydroquinazolinones as IRAP Inhibitors. ChemistryOpen 2020, 9, 325-337. [CrossRef] [PubMed]

13. Mpakali, A.; Saridakis, E.; Harlos, K.; Zhao, Y.; Kokkala, P.; Georgiadis, D.; Giastas, P.; Papakyriakou, A.; Stratikos, E. LigandInduced Conformational Change of Insulin-Regulated Aminopeptidase: Insights on Catalytic Mechanism and Active Site Plasticity. J. Med. Chem. 2017, 60. [CrossRef]

14. Mpakali, A.; Saridakis, E.; Harlos, K.; Zhao, Y.; Papakyriakou, A.; Kokkala, P.; Georgiadis, D.; Stratikos, E. Crystal Structure of Insulin-Regulated Aminopeptidase with Bound Substrate Analogue Provides Insight on Antigenic Epitope Precursor Recognition and Processing. J. Immunol. 2015, 195, 2842-2851. [CrossRef]

15. Giastas, P.; Neu, M.; Rowland, P.; Stratikos, E. High-Resolution Crystal Structure of Endoplasmic Reticulum Aminopeptidase 1 with Bound Phosphinic Transition-State Analogue Inhibitor. ACS Med. Chem. Lett. 2019, 10, 708-713. [CrossRef] [PubMed]

16. Liddle, J.; Hutchinson, J.P.; Kitchen, S.; Rowland, P.; Neu, M.; Cecconie, T.; Holmes, D.S.; Jones, E.; Korczynska, J.; Koumantou, D.; et al. Targeting the Regulatory Site of ER Aminopeptidase 1 Leads to the Discovery of a Natural Product Modulator of Antigen Presentation. J. Med. Chem. 2020, 63, 3348-3358. [CrossRef] [PubMed]

17. Burley, S.K.; Bhikadiya, C.; Bi, C.; Bittrich, S.; Chen, L.; Crichlow, G.V.; Christie, C.H.; Dalenberg, K.; Di Costanzo, L.; Duarte, J.M.; et al. RCSB Protein Data Bank: Powerful new tools for exploring 3D structures of biological macromolecules for basic and applied research and education in fundamental biology, biomedicine, biotechnology, bioengineering and energy sciences. Nucleic Acids Res. 2021, 49, D437-D451. [CrossRef] [PubMed]

18. Sterling, T.; Irwin, J.J. ZINC 15-Ligand Discovery for Everyone. J. Chem. Inf. Model. 2015, 55, 2324-2337. [CrossRef] [PubMed]

19. Baell, J.B.; Holloway, G.A. New substructure filters for removal of pan assay interference compounds (PAINS) from screening libraries and for their exclusion in bioassays. J. Med. Chem. 2010, 53, 2719-2740. [CrossRef]

20. Morris, G.M.; Goodsell, D.S.; Halliday, R.S.; Huey, R.; Hart, W.E.; Belew, R.K.; Olson, A.J. Automated docking using a Lamarckian genetic algorithm and an empirical binding free energy function. J. Comput. Chem. 1998, 19, 1639-1662. [CrossRef]

21. Trott, O.; Olson, A.J. AutoDock Vina: Improving the speed and accuracy of docking with a new scoring function, efficient optimization, and multithreading. J. Comput. Chem. 2010, 31, 455-461. [CrossRef] [PubMed]

22. Reeves, E.; Islam, Y.; James, E. ERAP1: A potential therapeutic target for a myriad of diseases. Expert Opin Ther Targets 2020, 24, 535-544. [CrossRef] [PubMed]

23. Genheden, S.; Ryde, U. The MM/PBSA and MM/GBSA methods to estimate ligand-binding affinities. Expert Opin. Drug Discov. 2015, 10, 449-461. [CrossRef] [PubMed]

24. Wang, C.; Greene, D.; Xiao, L.; Qi, R.; Luo, R. Recent developments and applications of the MMPBSA method. Front. Mol. Biosci. 2018, 4, 87. [CrossRef] [PubMed]

25. Weis, A.; Katebzadeh, K.; Söderhjelm, P.; Nilsson, I.; Ryde, U. Ligand affinities predicted with the MM/PBSA method: Dependence on the simulation method and the force field. J. Med. Chem. 2006, 49, 6596-6606. [CrossRef]

26. Daina, A.; Michielin, O.; Zoete, V. SwissADME: A free web tool to evaluate pharmacokinetics, drug-likeness and medicinal chemistry friendliness of small molecules. Sci. Rep. 2017, 7. [CrossRef]

27. Daina, A.; Michielin, O.; Zoete, V. ILOGP: A simple, robust, and efficient description of n-octanol/water partition coefficient for drug design using the GB/SA approach. J. Chem. Inf. Model. 2014, 54, 3284-3301. [CrossRef]

28. Zervoudi, E.; Papakyriakou, A.; Georgiadou, D.; Evnouchidou, I.; Gajda, A.; Poreba, M.; Salvesen, G.S.; Drag, M.; Hattori, A.; Swevers, L.; et al. Probing the S1 specificity pocket of the aminopeptidases that generate antigenic peptides. Biochem. J. 2011, 435, 411-420. [CrossRef]

29. Zervoudi, E.; Saridakis, E.; Birtley, J.R.J.R.R.; Seregin, S.S.S.S.; Reeves, E.; Kokkala, P.; Aldhamen, Y.A.Y.A.A.; Amalfitano, A.; Mavridis, I.M.I.M.M.; James, E.; et al. Rationally designed inhibitor targeting antigentrimming aminopeptidases enhances antigen presentation and cytotoxic T-cell responses. Proc. Natl. Acad. Sci. USA 2013, 110, 19890-19895. [CrossRef]

30. Papakyriakou, A.; Stratikos, E. The role of conformational dynamics in antigen trimming by intracellular aminopeptidases. Front. Immunol. 2017, 8. [CrossRef]

31. Rogi, T.; Tsujimoto, M.; Nakazato, H.; Mizutani, S.; Tomoda, Y. Human placental leucine aminopeptidase/oxytocinase. A new member of type II membrane-spanning zinc metallopeptidase family. J. Biol. Chem. 1996, 271, 56-61. [CrossRef] [PubMed]

32. Stockert, J.C.; Horobin, R.W.; Colombo, L.L.; Blázquez-Castro, A. Tetrazolium salts and formazan products in Cell Biology: Viability assessment, fluorescence imaging, and labeling perspectives. Acta Histochem. 2018, 120, 159-167. [CrossRef] 
33. Mpakali, A.; Saridakis, E.; Giastas, P.; Maben, Z.; Stern, L.J.; Larhed, M.; Hallberg, M.; Stratikos, E. Structural Basis of Inhibition of Insulin-Regulated Aminopeptidase by a Macrocyclic Peptidic Inhibitor. ACS Med. Chem. Lett. 2020. [CrossRef]

34. Webb, B.; Sali, A. Comparative Protein Structure Modeling Using MODELLER. Curr. Protoc. Protein Sci. 2016, 86, $291-2937$. [CrossRef]

35. Anandakrishnan, R.; Aguilar, B.; Onufriev, A. V H++ 3.0: Automating pK prediction and the preparation of biomolecular structures for atomistic molecular modeling and simulations. Nucleic Acids Res. 2012, 40, W537-W541. [CrossRef]

36. Morris, G.M.; Huey, R.; Lindstrom, W.; Sanner, M.F.; Belew, R.K.; Goodsell, D.S.; Olson, A.J. AutoDock4 and AutoDockTools4: Automated docking with selective receptor flexibility. J. Comput. Chem. 2009, 30, 2785-2791. [CrossRef]

37. Salomon-Ferrer, R.; Gotz, A.W.; Poole, D.; Le Grand, S.; Walker, R.C. Routine Microsecond Molecular Dynamics Simulations with AMBER on GPUs. 2. Explicit Solvent Particle Mesh Ewald. J. Chem. Theory Comput. 2013, 9, 3878-3888. [CrossRef] [PubMed]

38. Case, D.A.; Cheatham, T.E.; Darden, T.; Gohlke, H.; Luo, R.; Merz, K.M.; Onufriev, A.; Simmerling, C.; Wang, B.; Woods, R.J. The Amber biomolecular simulation programs. J. Comput. Chem. 2005, 26, 1668-1688. [CrossRef]

39. Maier, J.A.; Martinez, C.; Kasavajhala, K.; Wickstrom, L.; Hauser, K.E.; Simmerling, C. ff14SB: Improving the Accuracy of Protein Side Chain and Backbone Parameters from ff99SB. J. Chem. Theory Comput. 2015, 11, 3696-3713. [CrossRef]

40. Wang, J.M.; Wolf, R.M.; Caldwell, J.W.; Kollman, P.A.; Case, D.A. Development and testing of a general amber force field. J. Comput. Chem. 2004, 25, 1157-1174. [CrossRef]

41. Jakalian, A.; Jack, D.B.; Bayly, C.I. Fast, efficient generation of high-quality atomic charges. AM1-BCC model: II. Parameterization and validation. J. Comput. Chem. 2002, 23, 1623-1641. [CrossRef] [PubMed]

42. Stamogiannos, A.; Maben, Z.; Papakyriakou, A.; Mpakali, A.; Kokkala, P.; Georgiadis, D.; Stern, L.J.L.J.; Stratikos, E. Critical Role of Interdomain Interactions in the Conformational Change and Catalytic Mechanism of Endoplasmic Reticulum Aminopeptidase 1. Biochemistry 2017, 56. [CrossRef]

43. Giastas, P.; Mpakali, A.; Papakyriakou, A.; Lelis, A.; Kokkala, P.; Neu, M.; Rowland, P.; Liddle, J.; Georgiadis, D.; Stratikos, E. Mechanism for antigenic peptide selection by endoplasmic reticulum aminopeptidase 1. Proc. Natl. Acad. Sci. USA 2019, 116. [CrossRef]

44. Roe, D.R.; Cheatham, T.E. PTRAJ and CPPTRAJ: Software for Processing and Analysis of Molecular Dynamics Trajectory Data. J. Chem. Theory Comput. 2013, 9, 3084-3095. [CrossRef]

45. Kollman, P.A.; Massova, I.; Reyes, C.; Kuhn, B.; Huo, S.; Chong, L.; Lee, M.; Lee, T.; Duan, Y.; Wang, W.; et al. Calculating structures and free energies of complex molecules: Combining molecular mechanics and continuum models. Acc. Chem. Res. 2000, 33, 889-897. [CrossRef]

46. Wang, C.; Nguyen, P.H.; Pham, K.; Huynh, D.; Le, T.B.N.; Wang, H.; Ren, P.; Luo, R. Calculating protein-ligand binding affinities with MMPBSA: Method and error analysis. J. Comput. Chem. 2016, 37, 2436-2446. [CrossRef]

47. Foloppe, N.; Hubbard, R. Towards Predictive Ligand Design with Free-Energy Based Computational Methods? Curr. Med. Chem. 2006, 13, 3583-3608. [CrossRef]

48. Yang, T.; Wu, J.C.; Yan, C.; Wang, Y.; Luo, R.; Gonzales, M.B.; Dalby, K.N.; Ren, P. Virtual screening using molecular simulations. Proteins Struct. Funct. Bioinform. 2011, 79, 1940-1951. [CrossRef]

49. Tan, C.; Tan, Y.H.; Luo, R. Implicit nonpolar solvent models. J. Phys. Chem. B 2007, 111, 12263-12274. [CrossRef]

50. Tsui, V.; Case, D.A. Theory and applications of the generalized Born solvation model in macromolecular simulations. Biopolymers 2000, 56, 275-291. [CrossRef]

51. Connolly, M.L. Analytical molecular surface calculation. J. Appl. Crystallogr. 1983, 16, 548-558. [CrossRef]

52. Hawkins, G.D.; Cramer, C.J.; Truhlar, D.G. Parametrized models of aqueous free energies of solvation based on pairwise descreening of solute atomic charges from a dielectric medium. J. Phys. Chem. 1996, 100, 19824-19839. [CrossRef]

53. Weiser, J.; Shenkin, P.S.; Still, W.C. Approximate atomic surfaces from linear combinations of pairwise overlaps (LCPO). J. Comput. Chem. 1999, 20, 217-230. [CrossRef]

54. Onufriev, A.; Bashford, D.; Case, D.A. Exploring protein native states and large-scale conformational changes with a modified generalized born model. Proteins 2004, 55, 383-394. [CrossRef]

55. Tan, C.; Yang, L.; Luo, R. How well does Poisson-Boltzmann implicit solvent agree with explicit solvent? A quantitative analysis. J. Phys. Chem. B 2006, 110, 18680-18687. [CrossRef]

56. Mpakali, A.; Giastas, P.; Mathioudakis, N.; Mavridis, I.M.; Saridakis, E.; Stratikos, E. Structural basis for antigenic peptide recognition and processing by Endoplasmic reticulum (ER) aminopeptidase 2. J. Biol. Chem. 2015, 290, 26021-26032. [CrossRef] 